Collection SFN 8 (2007) 23-42

(C) EDP Sciences, Les Ulis

DOI: $10.1051 /$ sfn:2007004

\title{
Diffusion des neutrons polarisés en incidence rasante
}

\author{
E. Kentzinger
}

Institut für Festkörperforschung, Forschungszentrum Jülich, 52425 Jülich, Germany

\begin{abstract}
Résumé. La méthode de diffusion des neutrons polarisés en incidence rasante donne accès de manière nondestructive aux corrélations magnétiques latérales dans les systèmes couches minces, non seulement dans une région proche de la surface, mais aussi dans les couches enfouies. Dans cette revue, les deux types de géométries instrumentales et leurs différents domaines de longueur de corrélation latérale accessibles sont introduits. On insiste aussi sur l'importance de considérer la cohérence du faisceau de neutrons. Ensuite, les phénomènes de réflexion et réfraction des neutrons polarisés aux interfaces entre couches magnétiques et la section efficace de diffusion en incidence rasante dans le cadre de l'approximation de Born de l'onde distordue sont présentés. On montre alors trois exemples d'utilisation de cette méthode pour l'étude des corrélations magnétiques. Enfin, quelques développements futurs de la méthode sont discutés.
\end{abstract}

\section{INTRODUCTION}

Au cours des 20 dernières années, la possibilité de préparer des multicouches magnétiques, matériaux confinés à l'échelle nanométrique le long d'une dimension, a mené à l'émergence d'une classe de systèmes aux propriétés physiques extraordinaires, tant au plan fondamental que du point de vue des applications en technologie de l'information. Des effets physiques comme le couplage d'échange intercouches, la magnétorésistance géante, la magnétorésistance tunnel ou le retournant de l'aimantation stimulé par un courant électrique [1,2] ont mené à la possibilité d'utiliser non plus seulement la charge mais aussi le spin de l'électron pour le stockage, le transport et le traitement de l'information.

Pour une compréhension complète de ces propriétés, l'approximation dans laquelle les aimantations des couches sont latéralement homogènes ne tient généralement pas. Citons pour exemple le couplage d'échange à l'interface entre une couche ferromagnétique et une couche antiferromagnétique menant à un décalage sur l'axe des champs du cycle d'hystérésis et à une augmentation de la coercivité du matériau ferromagnétique. L'amplitude de ce décalage est mesurée deux ordres de grandeurs plus petite que celle attendue en prenant l'hypothèse que l'orientation des moments magnétiques atomiques n'est pas modifiée par l'introduction de l'interface et les modèles actuels insistent sur la présence de domaines magnétiques dans les deux types de couches [3,4,5].

D'autre part, le développement des méthodes de lithographie [6,7] et d'auto-organisation [8,9] a permis l'émergence de systèmes de plus basses dimensionnalités encore comme des arrangements de lignes (1D) ou de plots (0D). De nouveaux effets peuvent être attendus si la taille de l'élément ou la période de 1 arrangement considéré est plus petite que certaines grandeurs caractéristiques du matériau utilisé, comme la longueur d'onde des électrons au niveau de Fermi, la longueur de diffusion de spin des électrons, ou la largeur des parois de domaines [6,10]. Par exemple, le processus de renversement de l'aimantation est radicalement modifié dans une bulle magnétique de diamètre tel que la formation de parois de domaines est exclue. Typiquement, ces tailles se situent de quelques nanomètres à quelques dizaines de nanomètres.

Du point de vue des applications, les nanostructures magnétiques sont les composants ultimes de nouveaux composants spin-électroniques comme les mémoires vives magnétiques ou les matériaux d'enregistrement structurés. La course vers la miniaturisation de tels composants et la proximité de la limite superparamagnétique font que l'interaction magnétique entre ces composants devient un paramètre de plus en plus important qu'il faut comprendre et contrôler [11,12].

Les techniques les plus usuelles d'investigation des corrélations latérales dans les matériaux magnétiques structurés, comme la microscopie à force magnétique (MFM), la microscopie par effet Kerr, 
ou la microscopie électronique par photoémission $X$ (X-PEEM) sont des méthodes qui ne sondent le matériau qu'en surface ou très proche de la surface et peuvent même parfois modifier la configuration magnétique du système. C'est le cas par exemple en MFM sur matériau à très faible coercivité magnétique où le déplacement de la pointe au dessus de la surface peut entrâner des mouvements de parois de domaine.

La diffusion des neutrons polarisés en incidence rasante donne accès, de manière non-destructive et non-perturbatrice, aux corrélations dans le plan des couches non seulement dans la région toute proche de la surface mais aussi dans les couches enfouies. Cette détermination quantitative et résolue en profondeur du paramètre d'ordre et de ses corrélations à toutes les échelles de grandeurs requises fait des neutrons polarisés une sonde unique, même comparée aux techniques décrites plus haut ou à la diffusion résonante de rayons $X$.

Dans la section suivante, je décris les différentes géométries expérimentales et dans quel cas de la diffusion en incidence rasante peut être observée. Ceci dépend du volume de cohérence du faisceau de neutrons projeté sur la surface de l'échantillon comparé aux tailles des corrélations latérales. Comme les exemples donnés ici proviennent du magnétisme, $\mathrm{j}$ 'introduis ensuite les neutrons polarisés et quelques caractéristiques de la réflexion et de la transmission des neutrons polarisés aux interfaces entre matériaux magnétiques. Ensuite, la section efficace de diffusion en incidence rasante est présentée dans l'approximation de Born de l'onde distordue (DWBA) pour prendre en compte le fait que, aux petits angles d'incidence et de diffusion sondés ici, la surface projetée du volume de cohérence peut être relativement grande. Afin d'illustrer les différents concepts introduits, je donne ensuite des résultats expérimentaux obtenus dans trois systèmes. L'apparition ou non de diffusion en incidence rasante est reliée aux tailles relatives des longueurs de corrélation latérales et des longueurs obtenues par projection du volume de cohérence du faisceau de neutrons sur la surface. Enfin, je donne quelques indices sur ce que pourrait être le futur de la méthode.

\section{GÉOMÉTRIES EXPÉRIMENTALES}

Le principe de la mesure est représenté sur la Figure 1. Les neutrons arrivent sur la surface de l'échantillon avec le vecteur d'onde $k_{i}$ sous l'angle d'incidence rasante $\alpha_{i}$ et sont détectés avec le vecteur d'onde $k_{f}$ dont la direction est définie par l'angle $\alpha_{\mathbf{f}}$ dans le plan parallèle au plan d'incidence (défini par $k_{i}$ et la normale à la surface de l'échantillon) et par l'angle $\varphi$ dans le plan perpendiculaire. Soit $z$ l'axe engendré par la normale à la surface, $x$ l'axe donné par l'intersection du plan d'incidence avec la surface et $y$ l'axe perpendiculaire à $x$ et à $z$. Soit $Q=k_{f}-k_{i}$ le vecteur d'onde de diffusion et $Q_{/ /}=\left(Q_{x}, Q_{y}\right)$ sa composante dans le plan des couches. Nous restreindrons la discussion à la diffusion élastique, c'est à dire $k_{f}=k_{i}=2 \pi / \lambda$ où $\lambda$ est la longueur d'onde de la radiation.

Si l'échantillon reste invariant par une translation dans le plan des couches, alors de l'intensité ne peut-être observée que dans la direction spéculaire définie par $\alpha_{f}=\alpha_{i}$ et $\varphi=0$. Si l'échantillon présente des fluctuations latérales comme de la rugosité aux interfaces ou des domaines magnétiques, alors de la diffusion peut être observée dans les directions $\alpha_{f} \neq \alpha_{i}$ et $\varphi \neq 0$. En général la réflectivité spéculaire et la diffusion en incidence rasante sont observées simultanément. La réflectivité donne alors des informations sur le paramètre d'ordre moyenné sur les coordonnées latérales et la diffusion donne des informations sur les fluctuations autour de cette valeur moyenne.

Sur un réflectomètre, la collimation du faisceau incident est définie par une paire de fentes parallèles à l'axe $y$. Dans ce cas, le signal détecté est intégré le long de $\varphi$, la diffusion peut être discriminée de la réflectivité spéculaire le long de la ligne hors-spéculaire (en rouge sur la Figure 1) et les corrélations latérales sont sondées le long de la direction $x$. Sur un appareil de diffusion aux petits angles, la collimation incidente est définie par deux paires de fentes, une verticale et parallèle à l'axe $y, 1$ 'autre horizontale. Dans ce cas, $\varphi$ est aussi résolu, la diffusion peut être mesurée le long de la ligne de diffusion hors-spéculaire et la ligne de diffusion aux petits angles en incidence rasante (en vert sur la Figure 2), et les corrélations latérales sont sondées le long des deux directions $x$ et $y$. 
En prenant compte de la petitesse des angles $\alpha_{i}, \alpha_{f}$ et $\varphi$ sondés, le vecteur de diffusion $Q=k_{f}-k_{i}$ se projette de la manière suivante sur les trois axes de coordonnées :

$$
Q=\left\{\begin{array}{l}
Q_{x} \approx k \cdot\left(\alpha_{i}^{2}-\alpha_{f}^{2}-\varphi^{2}\right) / 2 \\
Q_{y} \approx k \cdot \varphi \\
Q_{z} \approx k \cdot\left(\alpha_{i}+\alpha_{f}\right)
\end{array}\right.
$$

Notons que puisque $Q_{x}$ est une combinaison linéaire de carrés de petits angles, que $Q_{y}$ est linéaire en $\varphi$ et que les angles sont du même ordre de grandeur, alors $Q_{x}$ est toujours bien plus petit que $Q_{y}$. Typiquement, on a $0.5 \mu m<2 \pi / Q_{x}<20 \mu m$ et $1 \mathrm{~nm}<2 \pi / Q_{y}<300 \mathrm{~nm}$. Il apparaît donc que la diffusion hors spéculaire et la diffusion aux petits angles en incidence rasante (GISANS) sondent deux gammes de longueur différentes. Comme le GISANS donne accès à l'échelle nanométrique, on observe actuellement une ruée vers cette méthode, en neutrons comme en rayons $X$.

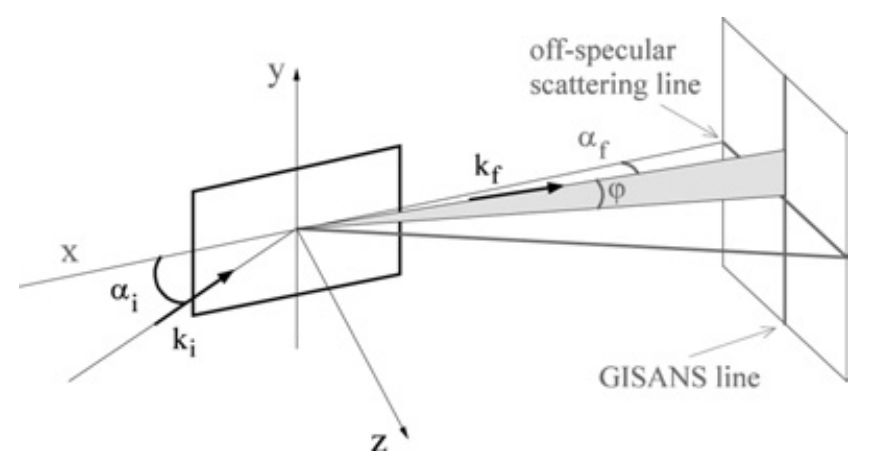

Figure 1. Les différentes géométries de diffusion en incidence rasante : diffusion hors spéculaire et diffusion aux petits angles en incidence rasante (GISANS).

Les mesures de réflectivité et de diffusion hors spéculaire sur un réflectomètre se représentent de la manière suivante (Figure 2(a)). Les intensités sont représentées en fonction de $\alpha_{i}$ et $\alpha_{f}$. Celles comptées selon la direction spéculaire $\left(Q_{x}=0\right)$ se trouvent le long de la diagonale principale d'équation $\alpha_{f}=\alpha_{i}$. En dehors de cette diagonale, la diffusion hors spéculaire est mesurée et les corrélations latérales selon la direction $x$ sont sondées $\left(Q_{x} \neq 0\right)$. Notons aussi que, le long de lignes perpendiculaires à cette diagonale et d'équation $\alpha_{i}+\alpha_{f}=$ constante, on sonde les corrélations selon $x$ à $Q_{z}$ constant (cf. équation 1). Un réflectomètre optimisé pour la mesure de la diffusion hors spéculaire (en neutrons polarisés) est le réflectomètre HADAS de Jülich [13] (appelé maintenant TREFF à FRM-II).

a)

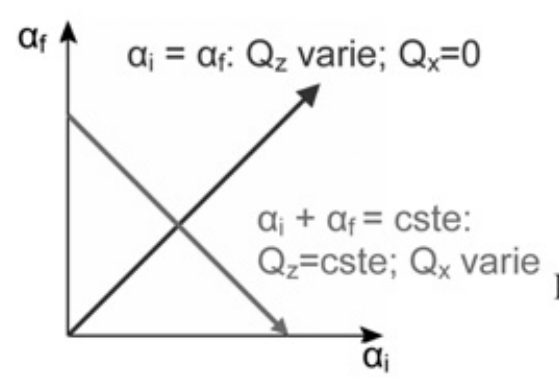

b)

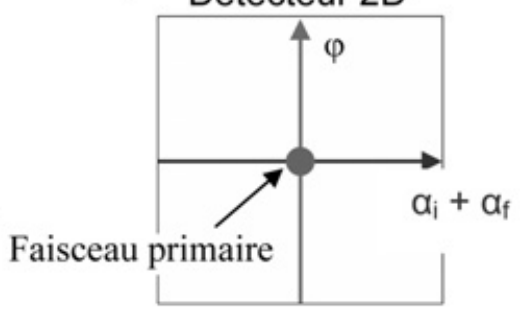

Figure 2. Représentation des données (a) en diffusion hors spéculaire et (b) en GISANS. 
Les mesures de GISANS se font sur un appareil de diffusion aux petits angles avec l'échantillon couche mince installé sur un goniomètre permettant de faire varier précisément l'angle d'incidence $\alpha_{i}$ du faisceau de neutrons. Pour un angle d'incidence et une distance échantillon-détecteur bien définis, l'intensité collectée sur le détecteur 2D donne la diffusion en fonction de $\alpha_{i}+\alpha_{f}$ et $\varphi$ (Figure 2(b)). Puisque $Q_{y}=k . \varphi$, l'axe $\varphi$ donne directement les corrélations suivant l'axe $y$. Le pic de réflectivité spéculaire $\left(\alpha_{f}=\alpha_{i}\right)$ se trouve centré autour de la coordonnée $\left(\alpha_{i}+\alpha_{f}=2 \alpha_{i}, \varphi=0\right)$.

\section{COHÉRENCE DU FAISCEAU DE NEUTRONS}

Une quantité importante à considérer dans l'interprétation et la simulation des mesures de réflectivité spéculaire et de diffusion en incidence rasante est le volume de cohérence du faisceau de neutrons, car sa projection $S_{c o h}$ sur la surface de l'échantillon, bien que parfois assez grande pour inclure des effets de diffusion multiple, reste toujours bien plus petite que la surface (illuminée) de l'échantillon. $S_{c o h}=L_{c o h}^{x} \cdot L_{c o h}^{y}$ est reliée aux résolutions $\delta Q_{x}$ et $\delta Q_{y}$ des composantes dans le plan du transfert de moment : $L_{c o h}^{x} \propto 1 / \delta Q_{x}$ et $L_{c o h}^{y} \propto 1 / \delta Q_{y}$. Sur un réflectomètre $L_{c o h}^{x}$ varie de quelques $\mu \mathrm{m}$ à quelques $\mathrm{mm}$ et, puisque $\delta Q$ est relaxée, $L_{c o h}^{y}=0$. Sur un appareil de diffusion aux petits angles, $L_{c o h}^{x}$ est du même ordre de grandeur que sur un réflectomètre et $L_{c o h}^{y}$ varie de 10 à $500 \mathrm{~nm}$. $L_{c o h}^{x}$ dépend de la longueur d'onde $\lambda$, des angles $\alpha_{i}, \alpha_{f}$ et $\varphi$ et de leurs résolutions. $L_{c o h}^{y}$ dépend de $\varphi$, de $\lambda$ et de leurs résolutions. [16]

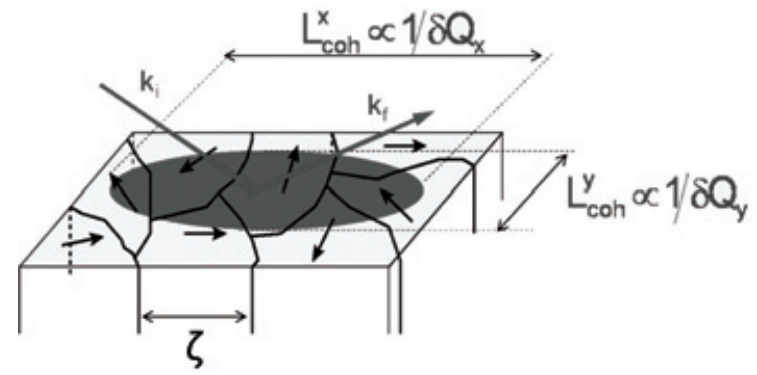

Figure 3. Projection du volume de cohérence du faisceau de neutrons sur la surface de l'échantillon.

$\mathrm{Si}$, à l'intérieur de $S_{c o h}$, l'échantillon peut être considéré comme homogène et à interfaces planes, il ne peut y avoir de transfert entre les composantes dans le plan des vecteurs d'onde $k_{i}$ et $k_{f}$ et de l'intensité n'est observée que le long de la direction spéculaire définie par $Q_{/ /}=0$ avec une précision donnée par $\delta Q_{/ /}$. La réflectivité mesurée est alors une moyenne sur les différentes réflectivités engendrées par les différentes surfaces de cohérence qui composent la surface de l'échantillon et pour lesquelles l'approximation homogène et plane tient. Ainsi, l'absence de diffusion en incidence rasante n'est pas toujours due au fait que l'échantillon est homogène sur toute sa surface. Si des fluctuations existent avec des longueurs de corrélation plus grandes que $L_{c o h}^{x}$ le long de la direction $x$ ou plus grandes que $L_{c o h}^{y}$ le long de la direction $y$, alors le signal mesuré reste purement spéculaire suivant la direction $x\left(Q_{x}=0\right)$ ou $y\left(Q_{y}=0\right)$ respectivement. Un tel cas est présenté dans la section 6.

$\mathrm{Si}$, au contraire, l'invariance du système par rapport à un déplacement latéral plus petit que $L_{c o h}^{x}$ ou $L_{c o h}^{y}$ est violée, un transfert de moment latéral peut avoir lieu et de l'intensité hors spéculaire peut être détectée, c'est-à-dire à $Q_{/ /} \neq 0$. La section efficace de diffusion est alors une moyenne des sections efficaces engendrées par les différentes surfaces de cohérence de l'échantillon.

En général le signal de diffusion en incidence rasante et la réflectivité spéculaire coexistent. La réflectivité provient de la variation en profondeur du potentiel d'interaction neutron-matière moyenné sur les coordonnées latérales et la diffusion en incidence rasante trouve son origine dans les fluctuations 
latérales autour de cette moyenne. Nous verrons un tel cas dans la section 8. Dans la section 7 nous verrons aussi qu'un signal de diffusion en incidence rasante pure (c.à.d. sans réflectivité) peut être observé à la position spéculaire.

\section{RÉFLEXION ET TRANSMISSION DES NEUTRONS POLARISÉS}

\subsection{Interaction neutron-matière magnétique}

Considérons ici une multicouche sans fluctuation latérale, le potentiel d'interaction neutron-matière n'ayant qu'une dépendance en profondeur. Dans chaque couche de la multicouche on suppose aussi que le potentiel n'a pas de dépendance en profondeur. Le neutron est une particule de spin $\frac{1}{2}$. Le potentiel d'interaction dans la couche 1 peut être séparé en deux parties, $\widehat{V}_{l}=V_{l}^{N} \widehat{1}+\widehat{V}_{l}^{M}$ où $V_{l}^{N}$ est le potentiel d'interaction nucléaire et $\widehat{V}_{l}^{M}=-\mu_{n} \widehat{\sigma} \cdot B_{l}$ est l'opérateur d'interaction dipôle magnétique entre l'opérateur moment dipolaire magnétique du neutron $\mu_{n} \widehat{\sigma}$ et le champ magnétique $B_{l}$ dans la couche 1 . est la matrice unité et $\widehat{\sigma}=\left(\widehat{\sigma}_{\perp}, \widehat{\sigma}_{z}, \widehat{\sigma}_{\prime \prime}\right)$ est le vecteur des matrices de Pauli :

$$
\widehat{\sigma}_{\perp}=\left(\begin{array}{cc}
0 & 1 \\
1 & 0
\end{array}\right) \widehat{\sigma}_{z}=\left(\begin{array}{cc}
0 & -i \\
i & 0
\end{array}\right) \widehat{\sigma}_{\prime \prime}=\left(\begin{array}{cc}
1 & 0 \\
0 & -1
\end{array}\right)
$$

«// » est l'axe de quantification du spin : les vecteurs propres $|+\rangle=\left(\begin{array}{l}1 \\ 0\end{array}\right)$ et $|-\rangle=\left(\begin{array}{l}0 \\ 1\end{array}\right)$ de $\widehat{\sigma}_{/ /}$ avec les valeurs propres +1 et -1 définissent respectivement les états de spin + et - . Le vecteur d'état du neutron dans l'espace des coordonnées et de spin est représenté par le spineur

$$
\left|\Psi_{l}(r)\right\rangle=\Psi_{l}^{+}(r)|+\rangle+\Psi_{l}^{-}(r)|-\rangle=\left(\begin{array}{c}
\Psi_{l}^{+}(r) \\
\Psi_{l}^{-}(r)
\end{array}\right)
$$

$\Psi_{l}^{+}(r)$ et $\Psi_{l}^{-}(r)$ sont les amplitudes de probabilité de trouver le spin dans les états + et -. Ces amplitudes dépendent de l'axe de quantification choisi.

L'opérateur d'interaction magnétique dans la couche 1 peut être décomposé en deux termes :

$$
\widehat{V}_{l}^{M}=-\mu_{n} \widehat{\sigma} \cdot\left[B_{0}+\mu_{0}(1-D) M_{l}\right]
$$

où $B_{0}$ est le champ externe appliqué, $M_{l}$ est l'aimantation dans la couche et $D$ est le facteur de désaimantation. Dans le cas d'une couche homogène aux dimensions latérales infinies $(1-D) M_{l}$ est égale à la composante de l'aimantation dans le plan [17].

L'opérateur potentiel d'interaction peut se réécrire sous la forme :

$$
\widehat{V}_{l}=\frac{2 \pi \hbar^{2}}{m}\left(\rho_{l}^{N} \widehat{1}+\rho_{l}^{M} \widehat{\sigma} \cdot b_{l}\right)
$$

où $\rho_{l}^{N}$ est la densité de longueur de diffusion nucléaire et $\rho_{l}^{M}$ est la densité de longueur de diffusion magnétique, introduite pour une comparaison facile des potentiels d'interaction nucléaire et magnétique. $b_{l}$ est le vecteur unité parallèle à $B_{l}$.

\subsection{Fonction d'onde du neutron}

Avant interaction avec l'échantillon le neutron peut être considéré comme une onde plane de vecteur d'onde $k_{0}$ et d'énergie $h^{2} k_{0}^{2} /(2 \mathrm{~m})$. Le spineur du neutron dans la couche 1 s'obtient par résolution de l'équation de Schrödinger à trois dimensions. Le potentiel d'interaction étant indépendant de la 
coordonnée latérale $\rho$ dans le plan des couches, il prend la forme $\left|\Psi_{l}(r)\right\rangle=e^{i \kappa \cdot \rho}\left|\Psi_{l}(z)\right\rangle$ où $\kappa$ est la composante dans le plan du vecteur d'onde dans la couche $1 k_{l}=k_{z, l} \cdot e_{z}+\kappa \cdot e_{\rho} \cdot \kappa$ est invariant d'une couche à l'autre. L'équation de Schrödinger à trois dimensions dans la couche 1 peut donc se réduire à deux équations à une dimension :

$$
\begin{aligned}
& \Psi_{l,+}^{\prime \prime}(z)+\left[k_{z, l}^{2}-4 \pi\left(\rho_{l}^{N}+\rho_{l}^{M} b_{l, / /}\right)\right] \Psi_{l,+}(z)-4 \pi \rho_{l}^{M} b_{l, \perp} \Psi_{l,-}(z)=0 \\
& \Psi_{l,-}^{\prime \prime}(z)+\left[k_{z, l}^{2}-4 \pi\left(\rho_{l}^{N}+\rho_{l}^{M} b_{l, / /}\right)\right] \Psi_{l,-}(z)-4 \pi \rho_{l}^{M} b_{l, \perp} \Psi_{l,+}(z)=0
\end{aligned}
$$

où $b_{l, / /}$ et $b_{l, \perp}$ sont les composantes de $b_{l}$ parallèle et perpendiculaire à l'axe de quantification.

Le spineur solution de l'équation de Schrödinger peut aussi s'écrire sous une forme indépendante du choix de l'axe de quantification :

$$
\left|\Psi_{l}(z)\right\rangle=\widehat{S}_{l}(z)\left|\Psi_{l}(0)\right\rangle
$$

où $\widehat{S}_{l}(z)$ est le propagateur dans la couche 1 avec :

$$
\widehat{S}_{l}(z)=e^{\hat{k}_{z, l}\left(z-z_{l-1}\right)} \widehat{t}_{l}+e^{-\widehat{i k}_{z, l}\left(z-z_{l-1}\right)} \widehat{r}_{l}
$$

où $z_{l-1}$ est la hauteur de l'interface entre les couches $l-1$ et 1 ( $z=0$ est l'interface avec l'air et $z$ est comptée positive vers le substrat) et où

$$
\widehat{k}_{z, l}=\sqrt{k_{z, 0}^{2}-4 \pi\left(\rho_{l}^{N} \widehat{1}+\rho_{l}^{M} \widehat{\sigma} \cdot b_{l}\right)}
$$

est la composante perpendiculaire du vecteur d'onde dans la couche 1 , avec $k_{z, o}=2 \pi \sin \alpha / \lambda$ où $\alpha$ est l'angle d'incidence. $\widehat{k}_{z, l}$ est un opérateur dans la base des états de spins. Comme toute fonction d'une combinaison linéaire de la matrice unité et des matrices de Pauli cet opérateur peut se réécrire comme une autre combinaison linéaire de la matrice unité et des matrices de Pauli $[18,19]$ :

$$
\begin{array}{r}
\widehat{k}_{z, 1}=\frac{1}{2}\left[\left(k_{z, 1}^{+}+k_{z, 1}^{-}\right) \hat{1}+\left(\widehat{\sigma} b_{1}\right) \cdot\left(k_{z, 1}^{+}-k_{z, 1}^{-}\right)\right] \text {où } \\
k_{z, 1}^{+}=\sqrt{k_{z, 0}^{2}-4 \pi\left(\rho_{1}^{N}+\rho_{1}^{M}\right)} \text { et } k_{z, 1}^{-}=\sqrt{k_{z, 0}^{2}-4 \pi\left(\rho_{1}^{N}-\rho_{1}^{M}\right)}
\end{array}
$$

sont les valeurs propres de $\widehat{k}_{z, l}$. Ainsi, si $b_{1}$ est choisit comme axe de quantification ( $\left.\widehat{\sigma} b_{l}=\widehat{\sigma}_{/ /}\right)$, la composante perpendiculaire du vecteur d'onde prend la valeur $k_{z, 1}^{+}$quand la projection du spin du neutron sur cet axe est $+1 / 2$ et $k_{z, 1}^{-}$quand sa projection est $-1 / 2$ (Figure 4 ).

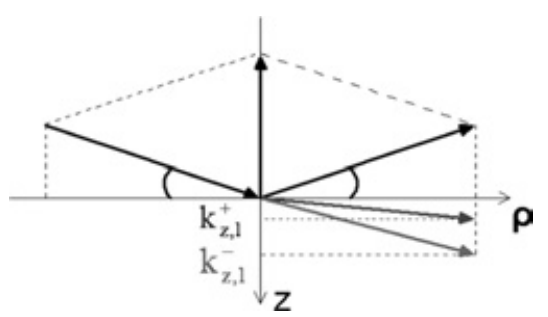

Figure 4. Dans un matériau ferromagnétique la composante perpendiculaire du vecteur d'onde dépend de la projection du spin sur l'axe de quantification. 
$\widehat{r}_{l}$ et $\widehat{t}_{l}$ dans (7) sont appelés coefficients de réflexion et de transmission dans la couche 1. Ils se déduisent récursivement en appliquant les conditions de continuité des $\left|\Psi_{l}(z)\right\rangle$ et de leurs dérivées premières aux interfaces. Si $N$ est le nombre de couches, le nombre d'interfaces est $N+1$ et les conditions de continuité conduisent à $2(N+1)$ équations. En prenant en compte le vide au dessus de la multicouche et le substrat et en considérant ces deux milieux comme semi-infinis, $2(N+2)$ coefficients de réflexion et de transmission doivent être déterminés. Deux autres équations sont obtenues en considérant que l'amplitude de transmission dans le vide est égale à la matrice unité $\left(\widehat{t}_{0}=\widehat{1}\right)$ et que, dans le substrat, il n'y a pas d'onde réfléchie $\left(\widehat{r}_{N+1}=\widehat{0}\right)$. Une résolution de ce système d'équation peut être trouvée dans [20].

$\widehat{r}_{l}$ et $\widehat{t}_{l}$ sont aussi des opérateurs dans la base des états de spin. Ils ont aussi deux valeurs propres différentes suivant la projection du spin sur l'axe de quantification. Il n'y a en général pas de formule analytique simple pour ces valeurs propres, sauf dans le cas d'un système constitué d'une seule interface entre le vide et un substrat magnétique. Dans ce cas, l'amplitude de réflexion dans l'air et l'amplitude de transmission dans le substrat se déduisent facilement des relations de continuité :

$$
\widehat{r}_{0}=\left(k_{z, 0}-\widehat{k}_{z, 1}\right) \cdot\left(k_{z, o}+\widehat{k}_{z, 1}\right)^{-1} \quad \text { et } \quad \widehat{t}_{1}=2 k_{z, 0} \cdot\left(k_{z, o}+\widehat{k}_{z, 1}\right)^{-1}
$$

Ces opérateurs sont aussi fonctions d'une combinaison linéaire de la matrice unité et des matrices de Pauli. On peut donc écrire :

$$
\widehat{r}_{0}=\frac{1}{2}\left[\left(r_{0}^{+}+r_{0}^{-}\right) \widehat{1}+\left(\widehat{\sigma} b_{1}\right) \cdot\left(r_{0}^{+}-r_{0}^{-}\right)\right] \quad \text { où } \quad r_{0}^{+}=\frac{k_{z, 0}-k_{z, 1}^{+}}{k_{z, o}+k_{z, 1}^{+}} \quad \text { et } \quad r_{0}^{-}=\frac{k_{z, 0}-k_{z, 1}^{-}}{k_{z, o}+k_{z, 1}^{-}}
$$

sont les valeurs propres de $\widehat{r}_{0}$, et

$$
\widehat{t}_{1}=\frac{1}{2}\left[\left(t_{1}^{+}+t_{1}^{-}\right) \hat{1}+\left(\widehat{\sigma} b_{1}\right) \cdot\left(t_{1}^{+}-t_{1}^{-}\right)\right] \quad \text { où } \quad t_{1}^{+}=\frac{2 k_{z, 0}}{k_{z, o}+k_{z, 1}^{+}} \quad \text { et } \quad t_{1}^{-}=\frac{2 k_{z, 0}}{k_{z, o}+k_{z, 1}^{-}}
$$

sont les valeurs propres de $\widehat{t}_{1}$. Les modules au carré de ces valeurs propres sont représentés sur la Figure 5. En dessous d'un angle d'incidence critique donné par $k_{z, 0}^{c+}=\sqrt{4 \pi\left(\rho_{1}^{N}+\rho_{1}^{M}\right)}$ et égal à $\alpha^{c+}=$ $\lambda \sqrt{\left(\rho_{1}^{N}+\rho_{1}^{M}\right) / \pi}, k_{z, 1}^{+}$dans la première équation (9) est imaginaire pur, l'onde dans le substrat est évanescente et $\left|r_{0}^{+}\right|^{2}=1$. On l'appelle l'angle critique de réflexion totale pour les neutrons de spin + . En $\alpha^{c+}$ le coefficient de transmission $t_{1}^{+}$est égal à 2 . Cette valeur provient de l'interférence constructive entre l'onde incidente et l'onde réfléchie. De la même manière, un angle critique de réflexion totale pour les neutrons de spin-est déduit de la deuxième équation (9).
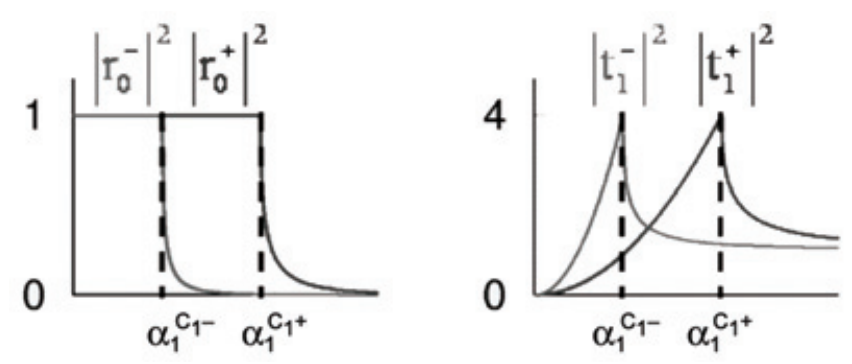

Figure 5. Modules au carré des valeurs propres des opérateurs de réflexion et de transmission à l'interface entre l'air et un substrat magnétique en fonction de l'angle d'incidence des neutrons. 


\subsection{Principe d'une mesure de réflectivité}

Le principe d'une mesure de réflectivité de neutrons polarisés est le suivant. On prépare d'abord un faisceau de neutrons avec tous les spins (ou presque, ceci dépend de l'efficacité du polariseur) dans l'état $|+\rangle$ ou avec tous les spins dans l'état $|+\rangle$ par rapport à un axe de quantification défini par le champ externe $B_{0}$. On laisse les neutrons interagir avec le substrat et on analyse ensuite le spin des neutrons par rapport au même axe de quantification. Quatre types de réflectivité peuvent être ainsi déterminés $R^{++}, R^{+-}, R^{-+}$et $R^{--}$. Ils se déduisent de l'opérateur de réflexion dans l'air $\widehat{r}_{0}$ :

$$
R^{++}=\left\langle\left|\left\langle+\left|\widehat{r}_{0}\right|+\right\rangle\right|^{2}\right\rangle, R^{+-}=\left\langle\left|\left\langle+\left|\widehat{r}_{0}\right|-\right\rangle\right|^{2}\right\rangle, R^{-+}=\left\langle\left|\left\langle-\left|\widehat{r}_{0}\right|+\right\rangle\right|^{2}\right\rangle \text { et } R^{--}=\left\langle\left|\left\langle-\left|\widehat{r}_{0}\right|-\right\rangle\right|^{2}\right\rangle
$$

Le signe $\langle\cdots\rangle$ dans chacune des égalités ci-dessus représente une moyenne des réflectivités sur les surfaces de cohérence du faisceau de neutrons illuminées par le faisceau de neutrons (cf. section 3). Une inspection de l'équation différentielle (6) qui régit la loi d'évolution des différentes composantes du spineur du neutron dans chacune des couches va nous permettre de déduire les propriétés de ces différentes réflectivités. On voit que seule la composante du champ magnétique perpendiculaire au champ externe $B_{0}\left(b_{l, \perp}\right)$ couple les amplitudes de probabilité de trouver le spin dans des états différents. La densité de longueur de diffusion nucléaire $\left(\rho_{l}^{N}\right)$ et la composante du champ magnétique parallèle à $B_{0}\left(b_{l, \mid /}\right)$ ne font appel dans (6) qu'à des états de spin identiques. Ainsi, dans une mesure de réflectivité de neutrons polarisés avec analyse du spin après interaction avec l'échantillon donne les réflectivités $R^{++}$et $R^{--}$«non - spin - flip » donnent accès au potentiel d'interaction nucléaire et à la composante de l'aimantation dans le plan des couches parallèle au champs appliqué et les réflectivités $R^{+-}$et $R^{-+}$ « spin - flip » donnent accès aux composantes de l'aimantation perpendiculaire à $B_{0}$. Par comparaison des intensités spin-flip et non-spin-flip on peut donc obtenir une information vectorielle sur la projection des aimantations dans le plan des couches.

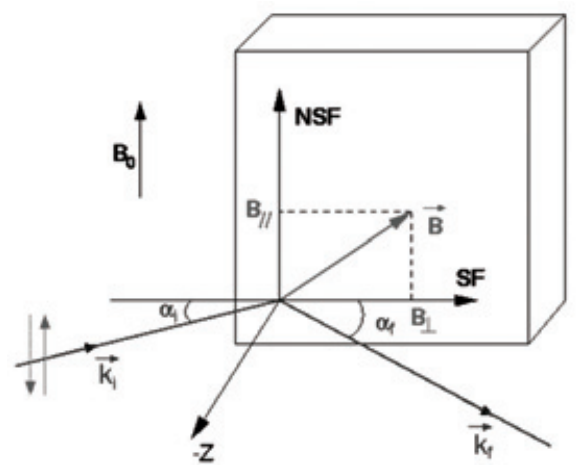

Figure 6. Règles de sélection de spin lors d'une mesure de réflectivité $\left(\alpha_{f}=\alpha_{i}\right)$ de neutrons polarisés. Les signaux non-spin-flip (NSF) permettent d'avoir accès à la densité de longueur de diffusion nucléaire et à la composante de l'aimantation parallèle au champ externe. Les signaux spin-flip (SF) permettent d'avoir accès à la composante de l'aimantation perpendiculaire au champ externe.

\section{SECTION EFFICACE DE DIFFUSION EN INCIDENCE RASANTE}

Si des fluctuations latérales de hauteur des interfaces, de longueur de diffusion nucléaire ou des champs magnétiques dans les couches existent avec des longueurs de corrélation plus petites que $L_{c o h}^{x}$ ou $L_{c o h}^{y}$, alors de la diffusion en incidence rasante peut être observée. En incidence rasante, $L_{c o h}^{x}$ et $L_{c o h}^{y}$ peuvent être relativement grandes (c.f. section 3) et les interactions avec les fluctuations peuvent mener à des 
processus de diffusion multiple au sein de la surface de cohérence. Dans ce cas une description de la section efficace de diffusion dans l'approximation de Born, qui suppose que chaque neutron n'est diffusé qu'une seule fois lors de son séjour dans l'échantillon, ne permet pas de reproduire les données mesurées.

Un moyen de remédier à ce problème est d'utiliser l'approximation de Born de l'onde distordue (en anglais : distorted wave Born approximation, DWBA) si l'hamiltonien du système peut être décomposé en la somme d'un hamiltonien de référence, qui peut être résolu exactement, plus un potentiel résiduel qui peut être traité en perturbation de l'hamiltonien de référence. Dans le cas d'une multicouche avec de la rugosité aux interfaces, l'hamiltonien de référence est celui du système avec des interfaces planes et dans le cas d'une multicouche avec des fluctuations micromagnétiques comme, par exemple, des domaines magnétiques, l'hamiltonien de référence dans chaque couche est obtenu en opérant une moyenne sur les aimantations.

Nous allons donner ici la section efficace dans le cas où les fluctuations ne sont pas des fluctuations d'interface, mais des fluctuations à l'intérieur de couches séparées par des interfaces planes. Dans ce cas, le potentiel d'interaction neutron-matière à l'intérieur de chaque couche 1 n'a qu'une dépendance le long le la coordonnée latérale $\rho$ dans le plan des couches et la décomposition du potentiel prend la forme :

$$
\widehat{V}_{l}(\rho)=\widehat{V}_{l}+\widehat{V}_{l}(\rho)
$$

où $\widehat{V}_{l}$ est le potentiel de référence et $\widehat{V}_{l}(\rho)$ est le potentiel résiduel décrivant les fluctuations, traité en perturbation et tel que $\left\langle\widehat{V}_{l}\right\rangle_{\rho}=0$, où le signe $\langle\cdots\rangle_{\rho}$ signifie la moyenne sur la coordonnée latérale $\rho$. Les $\widehat{V}_{l}$ donnent lieu à la réflectivité spéculaire (section 4) et les $\widehat{V}_{l}(\rho)$ à la diffusion (Figure 7)

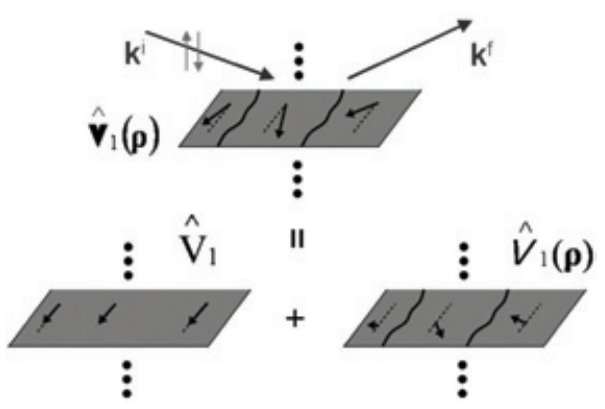

Figure 7. Décomposition du potentiel dans l'approximation de Born de l'onde distordue.

L'amplitude de diffusion du neutron incident avec le vecteur d'onde $k^{i}=\kappa^{i}+k_{z, 0}^{i} \cdot e_{z}$ et détecté avec le vecteur d'onde $k^{f}=\kappa^{f}+k_{z, 0}^{f} \cdot e_{z}$ s'écrit alors $[16,21]$ :

$$
F\left(k^{f}, k^{i}\right)=-\frac{m}{2 \pi \hbar^{2}} \int d r\left\langle\Psi^{f}\left(k^{f}, r\right)\left|\widehat{V}_{l}(\rho)\right| \Psi^{i}\left(k^{i}, r\right)\right\rangle
$$

Dans cette équation, le ket $\left|\Psi^{i}\left(k^{i}, r\right)\right\rangle$ et le bra $\left\langle\Psi^{f}\left(k^{f}, r\right)\right|$, tous les deux solutions de l'équation de Schrödinger pour le potentiel de référence, assument différentes conditions asymptotiques. $\left|\Psi^{i}\left(k^{i}, r\right)\right\rangle$ assume une onde plane incidente se rapprochant de l'échantillon en venant de la source, alors que $\left\langle\Psi^{f}\left(k^{f}, r\right)\right|$ assume une onde plane incidente se rapprochant de l'échantillon en venant du détecteur (Figure 8). De plus, contrairement à l'approximation de Born où le potentiel est pris entre un bra et un ket représentant des ondes planes avant interaction avec l'échantillon, en DWBA l'amplitude de 
diffusion est obtenue en prenant le potentiel résiduel entre un bra et un ket représentant des états du neutron distordus par le potentiel de référence.

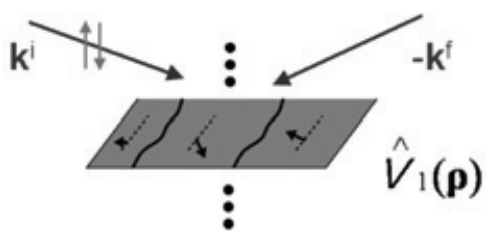

Figure 8. Représentation symbolique de l'amplitude de diffusion dans l'approximation de Born de l'onde distordue.

Dans chaque couche 1, le potentiel de référence est constant, le ket et le bra s'écrivent donc :

$$
\begin{aligned}
\left|\Psi_{l}^{i}\left(k^{i}, r\right)\right\rangle & =e^{i \kappa^{i} \rho} \cdot \widehat{S}_{l}^{i}(z) \cdot\left|\Psi_{l}^{i}(0)\right\rangle \text { avec } \widehat{S}_{l}^{i}(z)=e^{i \hat{k}_{z, l}^{i}\left(z-z_{l-1}\right)} \widehat{t}_{l}^{i}+e^{-i \widehat{k}_{z, l}^{i}\left(z-z_{l-1}\right) \widehat{r}_{l}^{i}} \\
\left\langle\Psi_{l}^{f}\left(k^{f}, r\right)\right| & =\left\langle\Psi_{l}^{f}(0)\right| \cdot \widehat{S}_{l}^{f}(z) \cdot e^{-i \kappa^{f} \rho} \text { avec } \widehat{S}_{l}^{i}(z)=\widehat{t}_{l}^{f} e^{\hat{k}_{z, l}^{f}\left(z-z_{l-1}\right)}+\widehat{r}_{l}^{f} e^{-\widehat{l}_{z, l}^{f}\left(z-z_{l-1}\right)}
\end{aligned}
$$

L'amplitude de diffusion s'exprime alors de la manière suivante :

$$
\begin{aligned}
F\left(Q_{/ /}, k_{z, 0}^{f}, k_{z, 0}^{i}\right) & =\sum_{l} \int d z\left\langle\Psi_{0}^{f}\left(k^{f}, 0\right)\left|\cdot \widehat{S}_{l}^{f}(z) \cdot \widehat{F}_{l}\left(Q_{/ /}\right) \cdot \widehat{S}_{l}^{i}(z) \cdot\right| \Psi_{0}^{i}\left(k^{i}, 0\right)\right\rangle \\
\text { où } \quad \widehat{F}_{l}\left(Q_{/ /}\right) & =-\frac{m}{2 \pi \hbar^{2}} \int d \rho e^{-i Q_{/ /}} \rho_{\widehat{V}_{l}}(\rho) \operatorname{avec} Q_{/ /} 3=\kappa^{f}-\kappa^{i}
\end{aligned}
$$

Ainsi, de manière similaire au calcul de l'amplitude de diffusion dans l'approximation de Born, le calcul de l'amplitude de diffusion dans l'approximation de Born de l'onde distordue inclue la transformée de Fourier latérale du potentiel résiduel. Les autres termes intervenant dans $F\left(Q_{/ /}, k_{z, 0}^{f}, k_{z, 0}^{i}\right)$ ne dépendent pas des fluctuations et ne font intervenir que le potentiel de référence. De la décomposition des opérateurs de propagation en combinaisons linéaires des opérateurs amplitude de réflexion et

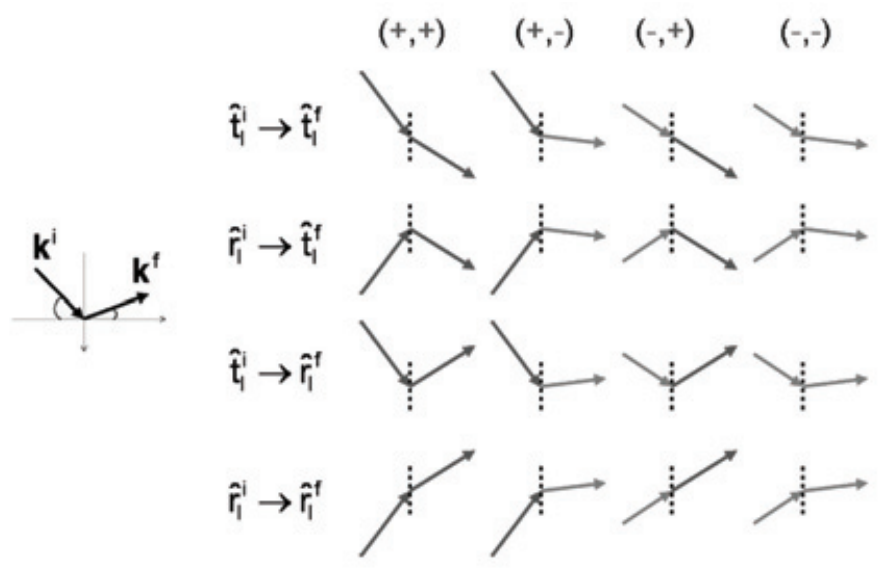

Figure 9. En DWBA, l'amplitude de diffusion dans le couche 1 magnétique se décompose en 16 termes. Les règles de sélections pour l'illumination de ces différents processus sont les mêmes que pour la réflectivité (section 4.3, Figure 5) en remplaçant les aimantations nettes et les densités d'amplitude de diffusion nucléaire par leurs fluctuations. 
amplitude de transmission et en considérant aussi le spin du neutron, l'amplitude de diffusion se décompose en 16 termes symboliquement représentés dans la Figure 4. Par exemple, le premier symbole en haut à gauche de la figure représente un neutron + incident transmis dans la couche 1 avec l'amplitude $t_{l}^{i+}$ (valeur propre de $\widehat{t}_{l}^{i}$ correspond à un neutron de spin + ), diffusé par une fluctuation de potentiel, puis transmis sans changement de projection du spin avec l'amplitude $t_{l}^{f+}$. La signification physique des quinze autres symboles se déduit de la même manière. Un processus de diffusion magnétique sans changement de la projection du spin apparaît dans le cas d'une fluctuation longitudinale du potentiel magnétique, c'est-à-dire lors d'une fluctuation de moment magnétique parallèle à l'axe de quantification et un processus de diffusion magnétique avec changement de spin apparaît dans le cas d'une fluctuation transversale, c'est-à-dire perpendiculaire à l'axe de quantification.

On peut alors calculer la section efficace de diffusion :

$$
\frac{d \sigma}{d \Omega}\left(Q_{/ /}, k_{z, 0}^{f}, k_{z, 0}^{i}\right)=\left\langle\left|F\left(Q_{/ /}, k_{z, 0}^{f}, k_{z, 0}^{i}\right)\right|^{2}\right\rangle
$$

où, comme dans la section 4.3 , le signe $\langle\cdots\rangle$ représente une moyenne des sections efficaces sur les différentes surfaces de cohérence illuminées par le faisceau. En insérant l'équation (18) dans (20) et en utilisant le théorème de Wiener-Khintchine qui relie le produit $\widehat{F}_{l}^{+}\left(Q_{\|}\right) \cdot \widehat{F}_{l}\left(Q_{\|}\right)$de deux transformées de Fourier latérales du type (19) dans les couches 1 et $\mathrm{m}$ à la transformée de Fourier latérale de la fonction de corrélation de paire entre les potentiels résiduels $\widehat{V}_{l}^{+}(\rho)$ et $\widehat{V}_{m}\left(\rho^{\prime}\right)$, on déduit que, de la même manière que dans l'approximation de Born, le calcul de la section efficace dans l'approximation de Born de l'onde distordue se réduit à la modélisation de fonctions de corrélations de paires. Les autres termes sont indépendants des fluctuations et ne font intervenir que le potentiel de référence [20,22]. Ils influent radicalement sur la section efficace à des angles $\alpha_{i}$ et $\alpha_{f}$ proches de la réflexion totale ou à des angles pour lesquels la structure multicouche moyenne engendre des pics de Bragg intenses.

\section{PROFIL D'AIMANTATION OSCILLANT DANS DES MULTICOUCHES À COUPLAGE DIPOLAIRE}

Je rapporte ici sur un état magnétique trouvé par réflectivité de neutrons polarisés dans des multicouches magnétiques à couplage dipolaire, montrant un profil en profondeur oscillant de l'aimantation. Ce profil provient d'une structure en domaines magnétiques corrélée, en accord avec des simulations micromagnétiques et des observations par microscopie par effet Kerr. Bien que cette structure en domaine existe, pas ou peu de diffusion hors-spéculaire en incidence rasante est observée. Ceci est dû à la grande taille des domaines, plus grande que la projection $L_{c o h}^{x}$ du volume de cohérence du faisceau de neutrons le long de l'axe $x$ défini dans la section 2 .

Différents types de couplage inter-couches peuvent exister dans les multicouches magnétiques composées de couches ferromagnétiques et non-magnétiques. Dans des tricouches constituées de deux couches métalliques ferromagnétiques saturées et séparées par une couche conductrice, un couplage d'échange oscillatoire de type Ruderman-Kittel-Kasuya-Yosida (RKKY) peut apparaitre [1,2] (voire aussi la section suivante). Dans les systèmes où la couche de séparation est un isolant électrique à interfaces planes, les interactions dipolaires sont dominantes. Elles favorisent une orientation relative antiparallèle des aimantations dans les couches adjacentes. Dans le cas de couches à interfaces rugueuses, un couplage inter couches de type Néel doit être considéré. En 1962, Louis Néel avait noté qu'il devrait $y$ avoir un couplage ferromagnétique entre films adjacents dû à la formation de dipôles magnétiques à l'interface induite par une ondulation morphologique corrélée [23]. Enfin, si l'on considère aussi la structure en domaines dans chacune des couches ferromagnétiques, un couplage magnétostatique peut apparaître, dû aux champs de fuite créés par les parois de domaines [24].

Le système étudié ici est une multicouche métal/isolant $\left.\mathrm{Co}_{80} \mathrm{Fe}_{20}\left(\mathrm{t}_{\mathrm{n}}\right) / \mathrm{Al}_{2} \mathrm{O}_{3}(3 \mathrm{~nm})\right]_{9}$. Pour des épaisseurs nominales $t_{n}$ plus petites que $1.6 \mathrm{~nm}$ le mode de croissance Volmer-Weber des couches de 
$\mathrm{CoFe}$ sur $\mathrm{Al}_{2} \mathrm{O}_{3}$ conduit à des couches discontinues de nanoparticules de $\mathrm{CoFe}$. A partir de $1.6 \mathrm{~nm}$ les nanoparticules deviennent suffisamment grosses pour assurer leur percolation dans le plan des couches. Dans ces multicouches l'arrangement vertical des amas de nanoparticules de couche à couche n'est pas aléatoire, menant à des profils de rugosité d'interface corrélés en profondeur, en accord avec des mesures de réflectivité et diffusion diffuse aux petits angles de rayons $X$ [25].

Pendant la croissance des échantillons, un faible champ magnétique appliqué parallèle au plan des couches induit un axe de facile aimantation dans le plan. Une mesure à $T=150 \mathrm{~K}$ du cycle d'hystérésis de l'aimantation avec le champ appliqué le long de l'axe de facile aimantation est représentée sur la Figure 10 (à gauche).

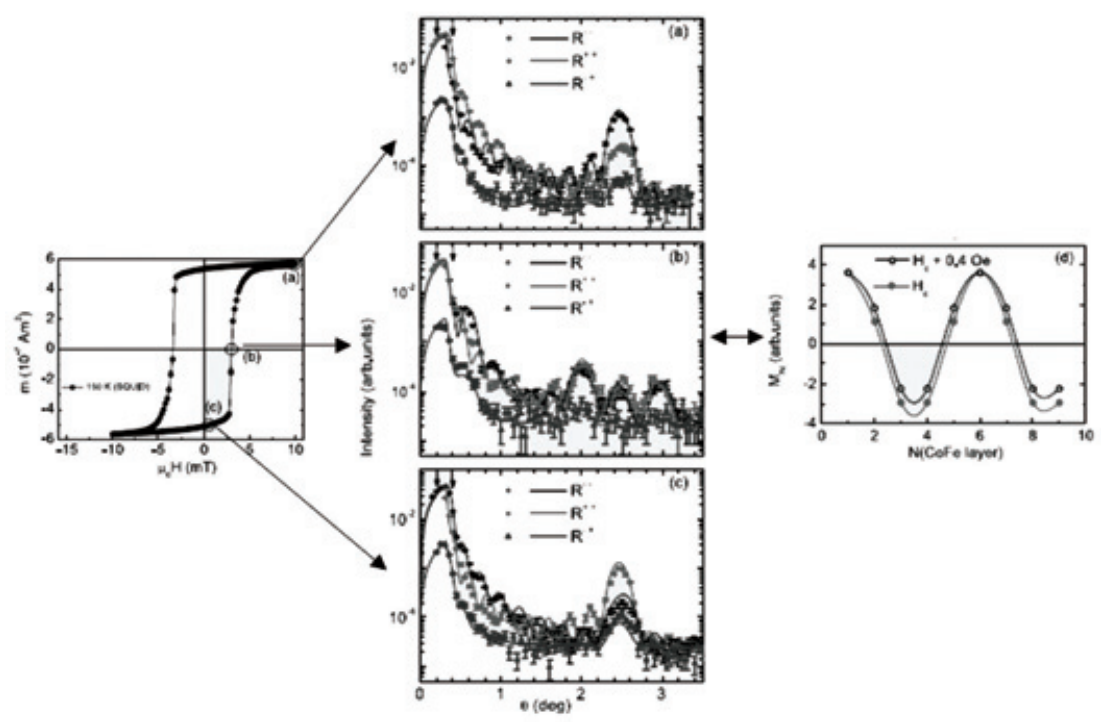

Figure 10. Mesures dans une multicouche $\left[\mathrm{Co}_{8} 0 \mathrm{Fe}_{2} \mathrm{O}(1.6 \mathrm{~nm}) / \mathrm{Al}_{2} \mathrm{O}_{3}(3 \mathrm{~nm})\right]_{9}$ (après [25]). Voire le texte pour les détails.

Le profil d'aimantation résolu en profondeur a été mesuré dans divers échantillons percolés $\left(t_{n} \geq\right.$ $1.6 \mathrm{~nm})$ par réflectivité et diffusion diffuse de neutrons polarisés sur le réflectomètre HADAS avec l'échantillon dans trois états magnétiques différents : à rémanence, au champ coercitif et à saturation. Dans tous les états, pas ou très peu de diffusion hors-spéculaire n'a été détectée. De ce fait, ne sont représentées dans la Figure 10 que les 4 mesures de réflectivité spéculaire avec analyse de polarisation $R^{++}, R^{+-}, R^{-+}$et $R^{--}$introduites dans la section 4.3. A rémanence et à saturation, les mesures sont caractéristiques d'un échantillon multicouche à aimantations identiques et parallèles, orientées antiparallèles et parallèles (respectivement) au champ appliqué (Figure 10, milieu). Les signaux spin-flip sont très peu intenses et sont bien reproduits en prenant en compte une polarisation du faisceau de neutrons imparfaite avant interaction avec l'échantillon et une efficacité de l'analyse de polarisation imparfaite aussi (94\% dans les deux cas), montrant l'absence d'une composante des aimantations perpendiculaire au champ appliqué. La non-dégénérescence des signaux non-spin-flip implique une composante nette de l'aimantation le long du champ appliqué. A saturation le signal $R^{++}\left(R^{--}\right)$est approximativement égal au signal $R^{--}$(resp. $R^{++}$) dans l'état rémanent sur toute la gamme d'angles d'incidence mesurée, montrant l'antiparallélisme des deux configurations magnétiques. Dans les deux états magnétiques, le premier pic de Bragg de la multicouche, dont la position est donnée par l'épaisseur d'une bicouche, est séparé du plateau de réflexion total par 7 franges de Kiessig en accord avec le nombre 9 de bicouches 
et dont l'espacement est donné par l'épaisseur totale de la multicouche. Au champ coercitif $\mu_{0} H_{C}$, on n'observe pas de signal spin-flip induit par l'échantillon, ni une dégénérescence des signaux non-spinflip sur toute la gamme d'angles mesurée, ce qui indique que le renversement des aimantations ne se produit ni par rotation des aimantations, ni par nucléation et croissance de domaines non-corrélés. De plus, deux pics satellites sont observés autour du pic de Bragg. Les données peuvent êtres simulées en supposant par un profil d'aimantation sinusoïdale de couche à couche de FeCo oscillant avec une période de 5 bicouches. Ce profil d'aimantation est représenté sur la Figure 10 (à droite, courbe rouge). Des mesures effectuées à $\mu_{0} H=\mu_{0} H C+0.04 \mathrm{mT}$ donnent lieu aussi à des satellites similaires autour du pic de Bragg à ceci près que les intensités $R^{++}$et $R^{--}$sont légèrement différentes qu' au champ coercitif. Le profil d'aimantation déduit est aussi modulé mais plus exactement une sinusoïde (Figure 10, à droite, courbe noire).

Des simulations micromagnétiques de ce système permettent de déduire ce profil d'aimantation d'un système de domaines magnétiques corrélés de couche à couche qui s'explique de la manière suivante. Les interactions dipolaires peuvent mener à des effets de frustration dans des multicouches magnétiques à aimantations dans le plan des couches. En général, les champs dipolaires de longue portée entre couches premières et deuxièmes voisines favorisent une orientation antiparallèle des aimantations. Puisque l'interaction entre couches premières voisines prévaut, un alignement antiparallèle sur toute la multicouche devrait apparaitre. Dans le cas de couches à interfaces rugueuses corrélées en profondeur, le couplage Néel inter couches de nature ferromagnétique préévaut et un alignement parallèle des aimantations sur toute la multicouche est stabilisé. Cependant, en appliquant un champ magnétique réverse, l'interaction ferromagnéétique est affaiblie par rapport à l'interaction antiferromagnétique dipolaire de second voisin. C'est pourquoi une modulation incommensurable de l'aimantation est observée dans les expériences comme dans les simulations.

Cette structure en domaines de l'état micromagnétique de l'échantillon est confirmée par des mesures de microscopie par effet Kerr longitudinal [26]. Ces mesures permettent aussi de déterminer les tailles des domaines, de l'ordre de la centaine de micromètres. Ceci explique aussi pourquoi pas ou peu de diffusion hors spéculaire n'a été observée sur HADAS. Cette taille moyenne des domaines se situe au dessus de la taille de la projection $L_{c o h}^{x}$ le long de l'axe $x$ sur la surface de l'échantillon du volume de cohérence du faisceau de neutrons, évaluée autour de $10 \mu \mathrm{m}$.

\section{DOMAINES MAGNÉTIQUES COLONNAIRES DANS UNE MULTICOUCHE À COUPLAGE D'ÉCHANGE ANTIFERROMAGNÉTIQUE}

Dans cette section je discute un système dans lequel la taille des domaines magnétiques est plus petite que la projection $L_{c o h}^{x}$ de la surface de cohérence du faisceau dans le plan des couches, donnant ainsi lieu à un fort signal de diffusion en incidence rasante. De plus, une partie du signal mesuré à la position spéculaire est d'origine diffusion en incidence rasante pure. La mesure de la diffusion hors spéculaire à $Q_{x} \neq 0$ est alors cruciale pour une description adéquate de l'ordre magnétique.

Le système présenté ici est une multicouche $[\operatorname{Co}(1.45 \mathrm{~nm}) / \mathrm{Cu}(1.02 \mathrm{~nm})]_{N=40}$ à fort couplage d'échange antiferromagnétique entre les couches de Co. Le but de l'étude était de déterminer la structure micromagnétique des couches de Co en fonction du champ magnétique appliqué perpendiculaire à l'axe de facile aimantation (Figure 11, en haut à droite) et de la corréler aux mesures macroscopiques de cycle d'aimantation et de magnétorésistance géante [27].

Ici nous n'insisterons pas sur la physique du système mais plutôt sur la méthode. Sur la Figure 11 (en bas à gauche) est représentée une mesure de réflectivité de neutrons polarisés lorsque l'échantillon est dans un état à aimantations antiparallèles, comme déterminé par les mesures macroscopiques de cycle d'aimantation et de magnétorésistance géante. La courbe de réflectivité montre un pic de Bragg nucléaire autour de $\alpha_{i}=96 \mathrm{mrad}$ dont la position sur l'axe des angles est donnée par la période de la multicouche, c.à.d. l'épaisseur d'une bicouche. Autour de la position moitié de cet angle $\left(\alpha_{i}=48 \mathrm{mrad}\right)$ 
se trouve le pic d'ordre antiferromagnétique, lié au doublement de la période magnétique par rapport à la période nucléaire. Au pic de Bragg nucléaire, la différence entre les intensités $R^{++}$et $R^{--}$est donnée par l'aimantation nette le long du champ appliqué et leurs dépendances en angle autour de cette position est bien reproduite par les simulations. L'absence de signal spin flip autour de cette position (à l'exception de celui induit par l'inefficacité des éléments de polarisation et inclus dans les simulations) indique l'absence d'une aimantation nette perpendiculaire au champ appliqué. Autour du pic de Bragg antiferromagnétique, des signaux de natures spin flip et non spin flip sont mesurés, avec une prépondérance des signaux spin-flip. Cependant, la simulation dans un modèle de réflectivités spéculaires (lignes sur la Figure 11 en bas à gauche) ne permet pas de reproduire les largeurs de ces pics suivant $\alpha_{i}$. Ceci est dû au fait que le signal mesuré à cette position est purement d'origine diffusion en incidence rasante.
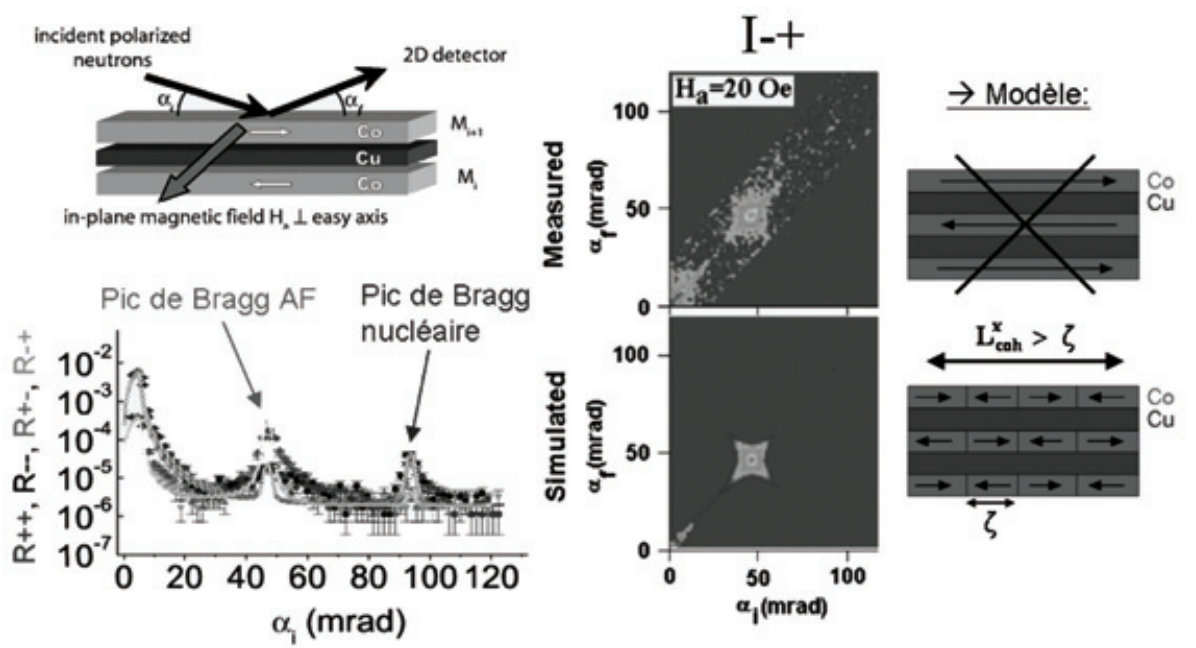

Figure 11. Réflectivité (en bas à gauche) et diffusion hors-spéculaire spin-flip (au milieu, en haut) de neutrons polarisés dans une multicouche $[\mathrm{Co}(1.45 \mathrm{~nm}) / \mathrm{Cu}(1.02 \mathrm{~nm})]_{N=40}$ à couplage d'échange inter couches antiferromagnétique mesurées sous un champ positif de 20 Oe après saturation négative de l'échantillon [27]. Sont représentés en haut à gauche la géométrie instrumentale, au milieu en bas une simulation de la diffusion hors spéculaire dans l'approximation de Born de l'onde distordue, et à droite le modèle micromagnétique déduit.

En effet, les mesures de diffusion hors spéculaires (Figure 11, au milieu en haut) montrent que les intensités mesurées au pic de Bragg AF le long de la ligne de réflectivité spéculaire sont en continuité avec les intensités mesurées à $\alpha_{i} \neq \alpha_{f}$ autour de ce pic, ceci dans les quatre canaux de spin $\mathrm{I}++, \mathrm{I}+-, \mathrm{I}-+$ et I- avec une prépondérance des intensités mesurées dans les canaux spin flip [28]. Ceci montre que l'état magnétique de l'échantillon est scindé en domaines, impliquant des fluctuations latérales corrélées de l'orientation des aimantations autour de leur orientation moyenne parallèle au champ appliqué. Ces fluctuations d'orientation induisent des fluctuations des composantes des aimantations perpendiculaires et parallèles au champ appliqué et produisent du diffus dans les canaux spin flip et non spin flip respectivement. Le fait que les intensités de diffusion diffuse restent concentrées autour du pic de Bragg montre une conformation colonnaire des domaines magnétiques (Figure 11, enbas à droite).

Les mesures de diffusion diffuse sont simulées dans l'approximation de Born de l'onde distordue $[27,28]$. Une telle simulation est représentée sur la Figure 11 (au milieu en bas). L'hamiltonien de référence est celui obtenu à partir du fit de la réflectivité autour du pic de Bragg nucléaire, c'est-àdire celui d'une multicouche avec une aimantation nette le long du champ appliqué. Le potentiel résiduel 
est obtenu en considérant des fluctuations corrélées de l'orientation des aimantations, induisant des fluctuations corrélées de leurs composantes perpendiculaires et parallèles au champ appliqué [20].

\section{CORRÉLATIONS LATÉRALES DANS UN SUPERMIROIR POLARISANT}

Dans cet exemple des signaux de diffusion en incidence rasante et de réflectivité spéculaire coexistent. Une investigation des corrélations latérales structurales et magnétiques a été effectuée à l'échelle micrométrique et à l'échelle nanométrique respectivement par diffusion diffuse hors spéculaire avec analyse de polarisation et par GISANS polarisé.

Les super-miroirs sont communément utilisés pour la polarisation de faisceaux de neutrons froids. Ils consistent en l'alternance de couches ferromagnétiques et non-magnétiques avec un gradient dans l'épaisseur des couches. Ce gradient permet d'étendre au delà de l'angle critique de réflexion totale la gamme d'angles pour laquelle la réflectivité des neutrons + est proche de 1. Les matériaux sont choisis de telle sorte que le contraste entre les potentiels d'interaction des couches ferromagnétiques et nonmagnétiques soit grand pour les neutrons de spin + et très petit pour les neutrons de spin -, assurant une réflectivité élevée pour les neutrons de spin + et une réflectivité quasi-nulle pour les neutrons de spin -. Dans un super-miroir polarisant et rémanent, une anisotropie macroscopique uniaxiale permet de garder une aimantation rémanente lorsque le champ magnétisant est éteint ou même légèrement renversé. Dans cet état, le super-miroir réfléchit bien les neutrons de spin - Cette propriété permet de construire un instrument fournissant des neutrons de spin + et - sans utiliser de spin-flipper [29]. Le super-miroir utilisé ici, acheté chez Swissneutronics, est constitué de 100 couches de $\mathrm{Fe}_{50} \mathrm{Co}_{48} \mathrm{~V}_{2}$ séparées par 100 couches de $\mathrm{TiN}_{\mathrm{x}}$.
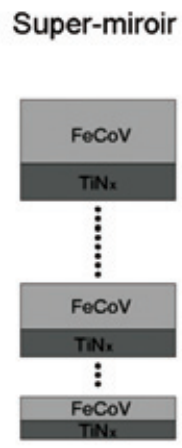

polarisant

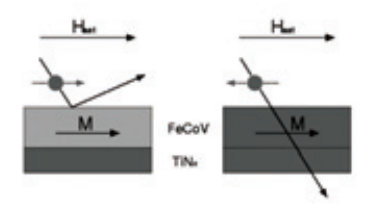

Figure 12. Caractéristiques d'un super-miroir polarisant rémanent.

Notre intérêt pour ce système réside dans la nanocristallinité des couches de $\mathrm{FeCoV}$ avec une orientation aléatoire des grains qui sont couplés avec leurs voisins par échange et qui ont des tailles plus petites que la longueur d'échange ferromagnétique, menant à une coercivité magnétique réduite [30]. Ceci, accompagné de la forte anisotropie uniaxiale mentionnée plus haut et de la forte aimantation à saturation des couches ferromagnétiques font de $\mathrm{FeCoV}$ un excellent candidat en tant que matériaux couche mince pour tête de lecture à haute fréquence [31]. Dans ce super-miroir, la taille latérale des grains augmente avec l'épaisseur des couches [32], menant à un accroissement de la coercivité magnétique de la couche la plus fine (et plus profonde) à la couche la plus épaisse (en surface) [29]. La question ici est de découvrir si cette évolution de la taille des grains se reflète dans une évolution de la microstructure magnétique et/ou dans une évolution du processus de renversement de l'aimantation des couches. C'est pourquoi une étude couche-par-couche du processus de renversement de l'aimantation a été entreprise par réflectométrie, diffusion hors-spéculaire de neutrons polarisés et GISANS polarisé. 


\subsection{Corrélations à l'échelle micrométrique par diffusion hors spéculaire [33]}

Sur la Figure 13 (à gauche) est représenté un exemple de mesure de réflectivité et diffusion hors spéculaire de neutrons polarisés avec analyse de polarisation effectuée sur le réflectomètre HADAS sur l'échantillon partiellement ré-aimanté obtenu par l'application d'un champ de $+4.5 \mathrm{mT}$ le long de l'axe de facile aimantation après saturation de l'échantillon sous champ négatif. La réflectivité spéculaire et la diffusion sont toutes les deux très polarisées en spin.

Dans l'état rémanent $\left(\mu_{0} H<2 \mathrm{mT}\right)$ toutes les aimantations dans les couches de FeCoV sont orientées antiparallèles au champ appliqué $\mu_{0} H$ : la réflectivité - - est intense et la réflectivité ++ est presque nulle. Dans l'état saturé $\left(\mu_{0} H<20 \mathrm{mT}\right)$ l'échantillon est saturé avec toutes les aimantations parallèles au champ, menant à une réflectivité ++ intense et à une réflectivité - - négligeable. A l'intérieur du plateau de réflectivité intense $\left(\alpha_{i}<20 \mathrm{mrad}\right)$, lorsque le champ est augmenté, la partie grands angles de la réflectivité - - perd son intensité et est remplacé par de la réflectivité ++. C'est une signature de l'augmentation de la coercivité des couches de $\mathrm{FeCoV}$ avec leur épaisseur. De l'analyse de la réflectivité spéculaire on déduit que, dans l'état présentement considéré (Figure 13) 88 des 100 couches de FeCoV ont retourné leurs aimantations parallèles au champ appliqué. L'absence de réflectivité spéculaire spin flip (en dehors de celle causée par l'efficacité limitée des éléments de polarisation) montre que le processus de renversement des aimantations ne se passe pas par rotation cohérente des aimantations mais par déplacement de parois de domaines.

\section{Mesures:}
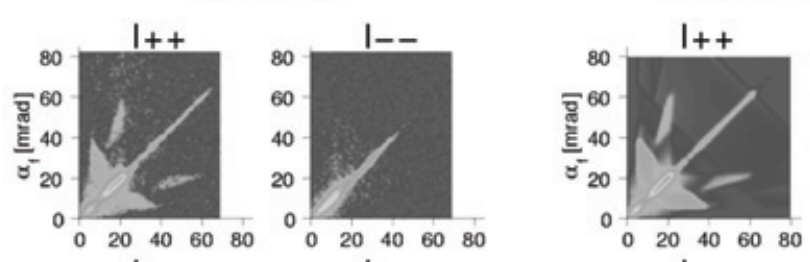

Simulations DWBA:
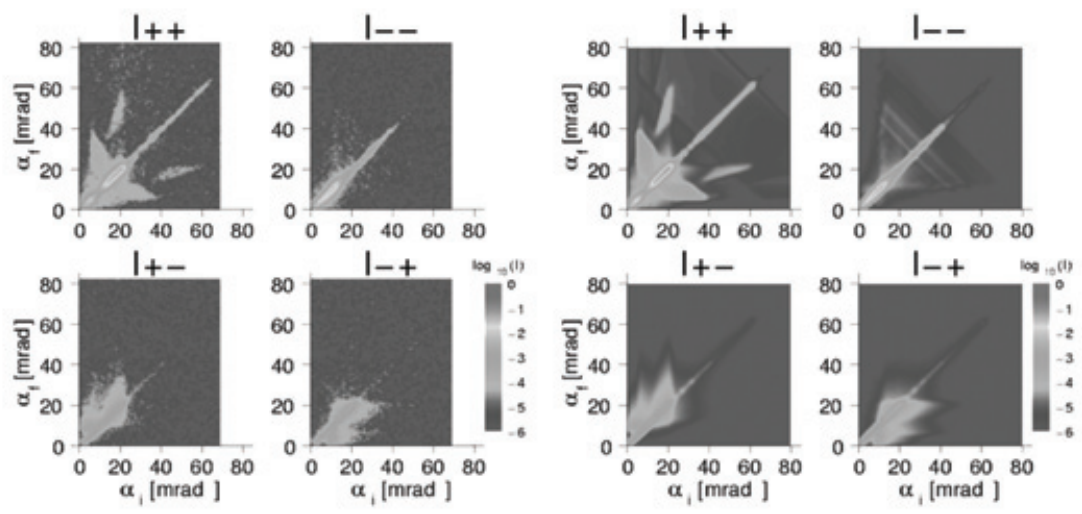

Figure 13. A gauche : Diffusion hors spéculaire de neutrons polarisés avec analyse de polarisation mesurée sous un champ de $4.5 \mathrm{mT}$ après saturation de l'échantillon dans un champ négatif. A droite : simulation des données dans l'approximation de Born de l'onde distordue (DWBA).

On note aussi une forte diffusion hors spéculaire dans les canaux non spin flip comme dans les canaux spin flip. Dans l'état rémanent, un fort signal diffus est observé dans le canal $I--$. Lorsque le champ est augmenté au-delà du champ rémanent et que les couches les plus fines commencent à renverser leurs aimantations, la disparition (l'apparition) de la réflectivité spéculaire -- (resp. ++) est accompagnée par la disparition (l'apparition) de la diffusion diffuse dans le même canal de spin. Ainsi, les intensités de diffusion diffuse dans les canaux non spin flip dépendent, de la même manière que la réflectivité spéculaire, du contraste entre couches $\mathrm{FeCoV}$ et $\mathrm{TiN}_{x}$ voisines. Elles sont donc dues à de la rugosité interfaciale. La comparaison des données avec des simulations DWBA montre que cette rugosité interfaciale est corrélée en profondeur. Cependant, le signal diffus ne s'organise pas «feuillesdeBragg » perpendiculaires à la ligne spéculaire comme c'est souvent le cas pour d'autres systèmes à rugosité interfaciale verticalement corrélée. L'explication est donnée dans [33]. 
On note aussi sur la Figure 13 des signaux hors spéculaires spin flip non négligeables. Ils sont dus à des fluctuations latérales corrélées de la composante de l'aimantation perpendiculaire au champ appliqué. Ces signaux n'ont pas tendance à s'organiser sous forme de «feuillesdeBragg ». Ils sont donc dus à des corrélations latérales magnétiques non corrélées en profondeur, ce que l'on peut attendre d'un système sans couplage inter couches.

Notons aussi l'asymétrie des signaux spin-flip par rapport à ligne spéculaire. Ceci peut s'expliquer en revenant à l'amplitude de diffusion dans l'approximation de Born de l'onde distordue (DWBA) présentée dans la section 5 (équation 18). L'amplitude de diffusion dans l'approximation de Born (équation 19) est multipliée à gauche et à droite par les opérateurs de propagation faisant intervenir les opérateurs de réflexion et de transmission. Ces opérateurs, qui ne dépendent que du potentiel de référence,ont des valeurs propres différentes suivant la projection du spin sur l'axe de quantification.

Voyons donc le canal $I-+$ de la Figure 13. Les neutrons arrivent sur l'échantillon sous l'angle d'incidence $\alpha_{i}$ et avec leur spin préparé - et sont détectés sous l'angle de sortie $\alpha_{f}$ avec le spin sélectionné +. Notons aussi que les réflectivités non spin flip qui permettent de déterminer l'état de référence sont prépondérantes dans des gammes d'angles différents suivant que l'on considère la réflectivité ++ ou la réflectivité -- . Ainsi, le signal diffus $I-+$ est non négligeable à des valeurs de $\alpha_{i}\left(\alpha_{f}\right)$ pour lesquelles la réflectivité - - (resp. ++) est non négligeable.

Notons finalement que tous les signaux hors spéculaires spin flip et non spin flip mesurés ici sont essentiellement déterminés par les coefficients de transmission et de réflexion et très peu par les corrélations. En effet, les simulations DWBA de la diffusion hors spéculaire non spin flip due à la rugosité interfaciale reproduisent toujours les données (à un facteur de calibration près) pourvu que la longueur de corrélation des rugosités soit prise plus petite que $0.1 \mu \mathrm{m}$. Aussi, les simulations DWBA de la diffusion hors spéculaire spin-flip ne permettent de déterminer qu'une longueur de corrélation magnétique moyennée sur toutes les couches et égale à $0.2 \pm 0.05 \mu \mathrm{m}$. Ceci est dû au fait que, dans la géométrie expérimentale présente, $Q_{x}$ est toujours trop petit par rapport aux inverses des longueurs de corrélation (voire section 2). Afin de déterminer ces longueurs de corrélation, la géométrie GISANS doit être utilisée.

\subsection{Corrélations à l'échelle nanométrique par GISANS polarisé [34]}

Les mesures de GISANS polarisé présentées Figure 14 ont été effectuées sur l'appareil de diffusion aux petits angles KWS-2 à Jülich avec l'échantillon dans le même état magnétique que dans la Figure 13. Dans la Figure 14 deux mesures GISANS sont présentées, une à une longueur de collimation de $8 \mathrm{~m}$ et une distance échantillon-détecteur de $3 \mathrm{~m}$ (C8-D3) et l'autre à une longueur de collimation et une distance échantillon-détecteur de 14 m (C14-D14). Chaque image montre les intensités collectées sur le détecteur 2D pour des neutrons incidents soit + , soit - . Les coordonnées verticale et horizontale ont été expliquées section 2, Figure 2 .

Dans la mesure à $\alpha_{i}=1.5^{\circ}$ et C8-D3, les intensités sont principalement concentrées autour de la position spéculaire $\left(\alpha_{i}+\alpha_{f}=3^{\circ}\right)$ dans les canaux $I+$ et $I-$ et le long de bandes perpendiculaires à l'axe $\alpha_{i}+\alpha_{f}$ et croisant cet axe à des valeurs de $\alpha_{i}+\alpha_{f}$ autour de $2.2^{\circ}, 3^{\circ}$ et $5.5^{\circ}$ dans le canal $I+$. Afin de comprendre ceci, les segments rouges ont été tracés sur les cartes de diffusion hors spéculaires à droite de la Figure 14 pour marquer le domaine de valeurs positives de $\alpha_{f}$ sondées par la mesure GISANS à la valeur de $\alpha_{i}$ considérée. On voit que les trois valeurs de $\alpha_{i}+\alpha_{f}$ considérées précédemment correspondent aux trois valeurs de $\alpha_{f}$ pour lesquelles le segment rouge croise la diffusion hors spéculaire générée par la rugosité d'interface dans le canal $I++$. De la largeur en $Q_{y}$ du signal GISANS on déduit une longueur de corrélation latérale de $15 \mathrm{~nm}$.

Les corrélations magnétiques, bien que de longueur de corrélation plus petite que le micromètre, ne sont pas résolues à C8-D3 (c.f. maximum local d'intensité autour de $\alpha_{i}+\alpha_{f}=1.8^{\circ}$ et $Q_{y}=0$ dans le canal $I-$ ) et doivent être investiguées avec une meilleure résolution en $Q_{y}$. Elles sont résolues dans la mesure à $\alpha_{i}=0.2^{\circ}$ et C14-D14 (en bas à gauche de la Figure 14). Dans cette mesure, outre la 
réflectivité spéculaire apparaissant à $\alpha_{i}+\alpha_{f}=0.4^{\circ}$ dans les deux canaux, les intensités sont localement maximisées autour de $\alpha_{i}+\alpha_{f}=0.9^{\circ}$ dans le canal $I+$. Dans le but de comprendre ceci, les segments jaunes sont tracés sur les cartes de diffusion hors spéculaire pour marquer le domaine de valeurs positives de $\alpha_{f}$ sondées par la mesure GISANS à la valeur de $\alpha_{i}$ considérée. On voit que la valeur de $\alpha_{i}+$ $\alpha_{f}$ considérée ici correspond à la valeur de $\alpha_{f}$ pour laquelle le segment jaune croise un maximum de diffusion hors spéculaire magnétique dans le canal $I+-$. De la largeur en $Q_{y}$ de ce pic GISANS magnétique, on déduit une longueur de corrélation des fluctuations de spin égale à $200 \mathrm{~nm}$. Notons ici que le domaine des valeurs de $\alpha_{f}$ positives sondées par la mesure de GISANS polarisé à C14D14 est petite, décroit avec $\alpha_{i}$ et se réduit à zéro pour $\alpha_{i}$ autour de $1.3^{\circ}$. Ceci ne permet donc pas d'effectuer une investigation des fluctuations magnétiques latérales dans tout le domaine des valeurs de $\alpha_{i}$ nécessaire pour une détermination complète des longueurs de corrélation magnétiques latérales sur toute la profondeur de la multicouche. Ce domaine des valeurs de $\alpha_{f}$ positives s'étend jusqu'à $2.5^{\circ}$, comme déduit des mesures de diffusion hors spéculaire.

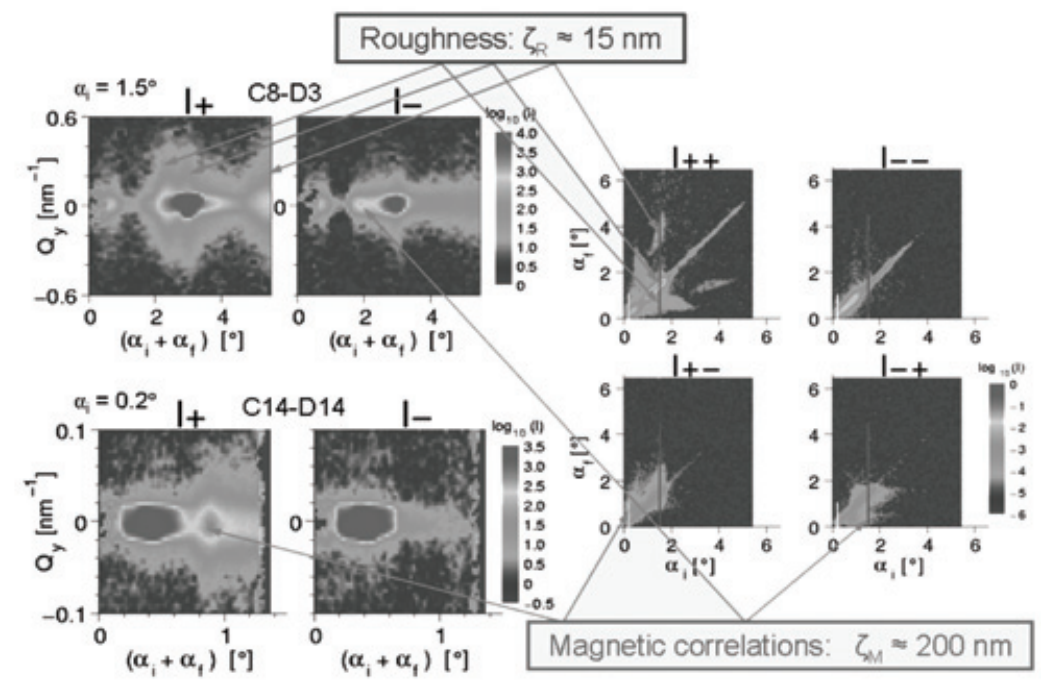

Figure 14. GISANS polarisé (à gauche) mesuré dans le même état magnétique que dans la Figure 13 et comparaison avec la réflectivité hors-spéculaire (à droite) [34]. Les mesures GISANS sont montrées en haut à gauche pour un angle d'incidence $\alpha_{i}=1.5^{\circ}$, une longueur de collimation de $8 \mathrm{~m}$ et une distance échantillon-détecteur de $3 \mathrm{~m}$ (C8-D3) et, en bas à gauche, pour un angle d'incidence $\alpha i=0.2^{\circ}$, une longueur de collimation et une distance échantillon-détecteur de $14 \mathrm{~m}$ (C14-D14). Pour ces deux géométries, les intensités ont été enregistrées avec un faisceau de neutrons incident préparé avec les spins préparés $+(I+)$ et $-(I-)$. Voire le texte pour la signification des segments rouge et jaune sur les cartes de diffusion hors spéculaires.

\section{CONCLUSIONS ET PERSPECTIVES}

La diffusion des neutrons polarisés en incidence rasante est une méthode très intéressante pour l'étude de l'ordre magnétique latéral dans les couches minces puisqu'il donne accès de manière non-destructive aux corrélations dans le plan non seulement dans la région proche de la surface mais aussi dans les couches enfouies. Cette détermination quantitative et résolue en profondeur du paramètre d'ordre et de ses corrélations à tous les ordres de grandeurs pertinents font des neutrons une sonde unique, même comparée aux méthodes que sont la microscopie à force magnétique (MFM), la microcopie électronique par photoémission $X$ ( $X$-PEEM) ou la diffraction magnétique résonante de rayons $X$. 
Des deux géométries expérimentales présentées dans la section 2, la GISANS est celle qui présente le potentiel scientifique le plus prometteur puisqu'elle permet d'accéder aux corrélations latérales à l'échelle nanométrique. Au sein du « JülichCenterforNeutronScience » fondé en 2006 [15], cette méthode est en cours d'implémentation sur le réflectomètre MARIA et les appareils de diffusion aux petits angles KWS-1 et KWS-3, tous les trois équipés d'analyse de polarisation. Les hauts flux de neutrons attendus permettront les mêmes types d'études nanomagnétiques que celles présentées plus haut mais sur des échantillons contenant moins de couches, comme c'est par exemple le cas des systèmes utilisés en spin-électronique. Ces hauts flux permettront aussi des études résolues en temps pour l'étude de la dynamique des aimantations à des échelles de temps descendant jusqu'à la $\mu s$.

\section{Remerciements}

De nombreux collègues ont contribué au travail présenté dans cette revue (voir les publications originales). Je tiens plus particulièrement à remercier Ulrich Rücker pour la qualité des données collectées sur le réflectomètre HADAS, Boris Toperverg qui m'a enseigné la manière de décrire la réflectivité et la diffusion de neutrons polarisés en incidence rasante, Henrich Frielinghaus et Dieter Richter pour leur soutien dans le développement de la méthode GISANS polarisé, Alexander Ioffe qui m'a enseigné les différentes manières de retourner le spin du neutron, et enfin Thomas Brückel pour l'impulsion qu'il a donnée à ce travail, et pour son intérêt et son soutien à toutes les étapes de son exécution.

\section{Références}

[1] Grünberg. P., J. Phys. : Condens. Matter 13, 7691 (2001).

[2] Bürgler, D. and Grünberg, P., in 32. IFF-Ferienkurses : Neue Materialien für die Informationstech$n i k$, Forschungszentrum Jülich, Series "Mater and Materials", Vol. 7 (2001).

[3] Nowak, U., Usadel, K. D., Keller, J., Miltényi, P., Beschoten, B., Güntherodt, G., Phys. Rev. B 66, 014430 (2002).

[4] Oldag, H., Scholl, A., Nolting, F., Renholz, E., Maat, S., Young, A. T., Carey, M., Stohr, J., Phys. Rev. Lett. 91, 017203 (2003).

[5] Roschin, I. V., Petracic, O., Morales, R., Li, Z.-P., Battle et, X., Schuller, I.K., Europhys. Lett. 71, 297 (2005).

[6] Martin, J. I., Nogues, J., Liu, K., Vincent et, J. L., Schuller, I. K., J. Magn. Magn. Mater. 256, 449 (2003).

[7] Ross, C. A., Harani, S., Castano, F. J., Hao, Y., Hwang, M., Shima, M., Cheng, J. Y., Vogeli, B., Farhoud, M., Walsh et, M., Smith, H. I., J. Appl. Phys. 91, 6848 (2002).

[8] Sun, S., Murray, C. B., Weller, D., Folks et L., Moser, A., Science 287, 1989 (2000).

[9] Cheng, J. Y., Jung et, W., Ross, C. A., Phys. Rev. B 70, 064417 (2004).

[10] Skomski, R., J. Phys. Condens. Matter 15, R841 (2003).

[11] Plumer, M., van Ek, J., Weller, D., The Physics of Ultra-high Density Magnetic Recording, Springer (2001).

[12] http://www.hitachigst.com/hdd/research/recording_head/pr/Perpendicular Animation.html.

[13] http://www.fz-juelich.de/iff/wns_hadas.

[14] http://www.fz-juelich.de/iff/wns_kws2.

[15] http://www.jens.info.

[16] Toperverg, B. P., "Polarized Neutron Reflection and Off-Specular Scattering" dans Polarized Neutron Scattering, Forschungszentrum Jülich, Series "Mater and Materials", Vol. 12 (2002).

[17] Fermon, C., Physica B 213 \& 214, 910 (1995).

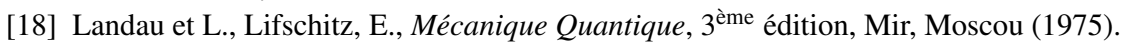


[19] Schärpf, O., "Polarization Elements" dans Polarized Neutron Scattering, Forschungszentrum Jülich, Series "Matter and Materials", Vol. 12 (2002).

[20] Kentzinger, E., Rücker, U., Toperverg, B., Physica B 335, 82 (2003).

[21] Toperverg, B. P., Physica B 297, 160 (2001).

[22] Toperverg, B. P., Appl. Phys. A 74, S1560-S1562 (2002).

[23] Néel, L., Acad. Sci., Paris, C.R. 255, 1676 (1962).

[24] Lew, W. S., Li, S. P., lopez-Diaz, L., Hatton, D. C., Bland, J. A. C., Phys. Rev. Lett. 90, 217201 (2003).

[25] Bedanta, S., Kentzinger, E., Petracic, O., Kleemann, W., Rücker, U., Brückel, Th., Paul, A., Cardoso, S., Freitas, P. P., Phys. Rev. B 74, 054426 (2006).

[26] Bedanta, S., Kentzinger, E., Petracic, O., Kleemann, W., Rücker, U., Paul, A., Brückel, Th., Cardoso, S., Freitas P. P., Physica B, in press (2007).

[27] Paul, A., Kentzinger, E., Rücker, U., Bürgler, D. E., Brückel, Th., Phys. Rev. B 73, 094441 (2006).

[28] Paul, A., Kentzinger, E., Rücker, U., Bürgler, D. E., Grünberg, P., Physica B 356, 31 (2005).

[29] Böni, P., Clemens, D., Senthil Kumar, M., Tixier, S., Physica B 241-243, 1060 (1998).

[30] Herzer, G., Magn. J., Magn. Mater. 112, 258 (1992).

[31] Contreras, M. C., Calleja, J. F., Matarranz, R., Presa, B., Corrales, J. A., Pan, G., J. Appl. Phys. 99, 8 F110 (2006).

[32] Senthil Kumar, M., Böni, P., J. Appl. Phys. 91, 3750 (2002).

[33] Kentzinger, E., Rücker, U., Toperverg, B., Brückel, Th., Ott, F., en préparation.

[34] Kentzinger, E., Frielinghaus, H., Rücker, U., Ioffe, A., Richter, D., Brückel, Th., Physica B (2007). 